\title{
Orthogeodesic Point-Set Embedding of Trees ${ }^{\star}$
}

\author{
Emilio Di Giacomo ${ }^{1}$, Fabrizio Frati ${ }^{2,3, \star \star}$, Radoslav Fulek ${ }^{2, \star \star}$, \\ Luca Grilli ${ }^{1}$, and Marcus Krug ${ }^{4}$
}

1 Dip. di Ingegneria Elettronica e dell'Informazione, Universitá degli Studi di Perugia, Italy \{digiacomo,grilli\}@diei.unipg.it

2 School of Basic Sciences, École Polytechnique Fédérale de Lausanne, Switzerland

\{fabrizio.frati,radoslav.fulek\}@epfl.ch

3 School of Information Technologies, University of Sydney

4 Institute of Theoretical Informatics, Karlsruhe Institute of Technology, Germany

marcus.krug@kit.edu

\begin{abstract}
Let $S$ be a set of $N$ grid points in the plane, and let $G$ a graph with $n$ vertices $(n \leq N)$. An orthogeodesic point-set embedding of $G$ on $S$ is a drawing of $G$ such that each vertex is drawn as a point of $S$ and each edge is an orthogonal chain with bends on grid points whose length is equal to the Manhattan distance. We study the following problem. Given a family of trees $\mathcal{F}$ what is the minimum value $f(n)$ such that every $n$-vertex tree in $\mathcal{F}$ admits an orthogeodesic point-set embedding on every grid-point set of size $f(n)$ ? We provide polynomial upper bounds on $f(n)$ for both planar and non-planar orthogeodesic point-set embeddings as well as for the case when edges are required to be $L$-shaped chains.
\end{abstract}

\section{Introduction}

Let $S$ be a set of $N$ points in the plane, and let $G$ be an $n$-vertex graph such that $n \leq N$. A point-set embedding of $G$ on $S$ is a drawing of $G$ such that each vertex is drawn as a point of $S$. Point-set embeddings are a classical subject of investigation in graph drawing from both an algorithmic and a combinatorial point of view. Different types of point-set embeddings have been defined depending on the desired type of drawing.

Several algorithmic results are known on point-set embeddings in which edges are required to be straight-line segments. Deciding whether a planar graph admits a straightline planar point-set embedding on a given point set is an NP-complete problem [5], while straight-line planar point-set embeddings of trees [3] and outerplanar graphs [2] can be computed efficiently. From the combinatorial perspective, Gritzmann et al. [12] prove that an $n$-vertex planar graph admits a straight-line planar point-set embedding on every set of $n$ points in general position if and only if it is outerplanar. Kaufmann and Wiese show that every $n$-vertex planar graph admits a polyline planar point-set embedding on every set of $n$ points with at most 2 bends per edge [14]. Colored versions of planar polyline point-set embeddings have also been investigated [17]. Special research efforts have been devoted to study universal point sets for planar graphs. A point set $S$ is universal for a family $\mathcal{F}$ of graphs and for a type $\mathcal{D}$ of drawing if every

\footnotetext{
* Initiated during the "Bertinoro Workshop on Graph Drawing", Bertinoro, Italy, March 2011.

${ }^{\star \star}$ Supported by the Swiss National Science Foundation Grant No.200021-125287/1.
} 
Table 1. Upper bounds on the value $f(n)$ obtained in this paper

\begin{tabular}{|c|c|c|c|c|}
\hline & Planar L-Shaped & Non-Planar L-Shaped & Planar & Planar 2-spaced \\
\hline Caterpillars $\Delta=3$ & $n$ [Th. 8] & $n$ [Th. 8] & $n$ [Th. 8] & $n$ [Th.1] \\
\hline Trees $\Delta=3$ & $n^{2}-2 n+2$ [Th. 6 & $n$ [Th. 10$]^{a}$ & $n$ [Th. 3] & $n$ [Th. 1] \\
\hline Caterpillars $\Delta=4$ & $3 n-2$ [Th.7] & $n+1$ [Th. 11] & $\lfloor 1.5 n\rfloor[$ Th. 4] & $n$ [Th. 1] \\
\hline Trees $\Delta=4$ & $n^{2}-2 n+2[$ Th. 6 & $4 n-3$ [Th.9] & $4 n$ [Th.2] & $n$ [Th. 1] \\
\hline
\end{tabular}

${ }^{a}$ Fink et al. [10] have independently obtained this result.

graph in $\mathcal{F}$ admits a point-set embedding of type $\mathcal{D}$ on $S$. Every universal point set for straight-line planar drawings of planar graphs has size at least $1.235 \cdot n[15]$ while there exist universal point sets of size $\frac{8}{9} n^{2}$ [4]. Universal point sets of size $n$ exist for polyline drawings of planar graphs [9].

In this paper we study orthogeodesic point-set embeddings on the grid. Orthogeodesic point-set embeddings were introduced by Katz et al. [13] and require edges to be represented by orthogeodesic chains, i.e. by orthogonal chains whose total length is equal to the Manhattan distance between the endpoints. Since orthogeodesic chains correspond to shortest orthogonal connections in the $L_{1}$ metric, they can be considered as the counter part of straight lines in the $L_{2}$ metric. Katz et al. [13] considered orthogeodesic point-set embeddings from the algorithmic side and proved that it is NP-complete to decide whether an $n$-vertex planar graph with maximum degree 4 admits an orthogeodesic point-set embedding on $n$ points, while the problem can be solved efficiently for cycles. Katz et al. [13] also show that, if the mapping between vertices and points is given and the bends are required to be at grid points, then the problem is NP-complete even for matchings, while the problem is polynomial-time solvable if bends need not be at grid points. Bi-colored planar orthogeodesic point-set embeddings have been studied by Di Giacomo et al. [6].

We consider orthogeodesic point-set embeddings on the grid from the combinatorial point of view. Let $P$ be a set of grid points in the plane, i.e., $p=(i, j)$ with $i, j \in \mathbb{Z}$ for all $p \in P$. We write $x(p):=i$ and and $y(p):=j$. A set $P$ of grid points with $x(p) \neq x(q)$ and $y(p) \neq y(q)$ for all $p, q \in P$ with $p \neq q$ is called general. For different classes of trees $\mathcal{F}$ and different drawing styles $\mathcal{D}$ we study the value $f(n)$ such that every general pointset is universal for orthogeodesic point-set embeddings of all trees in $\mathcal{F}$ using $\mathcal{D}$. The restriction to general point sets is necessary since there are arbitrarily large point sets that are not universal for orthogeodesic point-set embeddings of trees, e.g., a set of collinear points. We consider both planar and non-planar orthogeodesic point-set embeddings as well as the case when edges can be arbitrary orthogeodesic chains or when are edges required to be $L$-shaped chains. An $L$-shaped chain is an orthogonal chain with only one bend, thus, it is an orthogeodesic chain with the minimum number of bends for general point sets. Table 1 summarizes our results.

The rest of the paper is organized as follows. In Sects. 2, 3, and 4 we study planar, planar $L$-shaped, and non-planar $L$-shaped orthogeodesic point-set embeddings, respectively. Sect. 5] concludes and lists some open problems. 


\section{Planar Orthogeodesic Pointset Embeddings}

In this section we consider planar orthogeodesic point-set embeddings of trees. First, we show that every tree with maximum degree 4 can be embedded on every general point set with $n$ points with at most two bends per edge, if we require that the horizontal and vertical distance of any two points is at least two. We call point sets with this property 2 -spaced. This implies that we can embed every tree with $n$ vertices on every general point set $P$ with $n$ points whose points are not horizontally or vertically aligned, if neither vertices nor bends are required to be grid points.

Theorem 1. Every tree with $n$ vertices and with maximum degree 4 admits a planar orthogeodesic point-set embedding on every general point set $P$ with $n$ points such that $\min \{|x(p)-x(q)|,|y(p)-y(q)|\} \geq 2$ for all $p, q \in P$ with $p \neq q$.

Proof: Let $T$ be any tree with $n$ vertices and maximum degree 4. Root $T$ at any node $r$ of degree at most 3 . We prove that $T$ admits a planar orthogeodesic point-set embedding on every general point set $P$ with $n$ points in which: (i) each edge has two bends and (ii) no edge intersects a half-line arbitrarily chosen among the two horizontal and two vertical half-lines starting at $r$.

The statement is trivially true for $n=1$. We inductively prove that $T$ admits the required embedding for the case that no edge may intersect the horizontal half-line starting at $r$ and directed rightward (the other constructions are analogous). Let $n_{1} \geq 0, n_{2} \geq 0$, and $n_{3} \geq 0$ denote the number of vertices in the subtrees $T_{1}, T_{2}$, and $T_{3}$ rooted at children $r_{1}, r_{2}$, and $r_{3}$ of the root $r$ of $T$, respectively. Refer to Fig. 1 Let $P_{1}$ denote the set of the $n_{1}$ bottommost points of $P$. Let $P_{2}$ denote the set of the $n_{2}$ leftmost points of $P \backslash P_{1}$. Let $p$ be the bottommost point of $P \backslash\left(P_{1} \cup P_{2}\right)$. Let $P_{3}=P \backslash\left(P_{1} \cup P_{2} \cup\{p\}\right)$. Embed $r$ on $p$. Inductively embed $T_{i}$ on $P_{i}(i=1,2,3)$ with no edge intersecting the vertical half-line starting at $r_{1}$ directed upward. Connect $r$ with $r_{1}$ by an orthogeodesic edge vertically attached

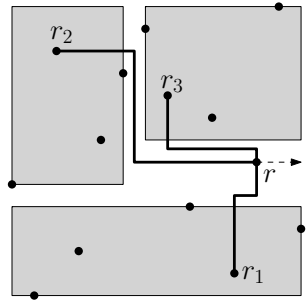

Fig. 1. Planar orthogeodesic point-set embedding of a tree on a general 2-spaced point set to $r$ and to $r_{1}$ and having an intermediate segment $s$ on the hori-

zontal line one unit above the top side of the bounding box of $P_{1}$. Connect $r$ with $r_{2}$ and $r_{3}$ analogously (see Fig. 1). It is easy to see that the constructed embedding is planar and that no edge intersects the horizontal half-line starting at $r$ and directed rightward. Since $\min \{|x(p)-x(q)|,|y(p)-y(q)|\} \geq 2$ for all $p, q \in P$ and since the intermediate segment $s$ occupies a grid line one unit above a point, this grid line does not contain any point from $P$.

As a consequence of Theorem 1 we obtain the following theorem for general point sets without the restriction on the horizontal and vertical distance of the points.

Theorem 2. Every tree with $n$ vertices and with maximum degree 4 admits a planar orthogeodesic point-set embedding on every general point set with $4 n$ points.

Proof: We prove that any set $P$ of $4 n$ points contains a subset of $n$ points such that no two points have a horizontal or vertical distance of less than two. The theorem then 


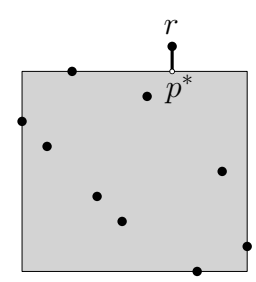

(a)

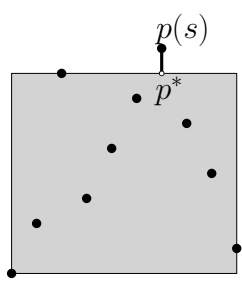

(b)

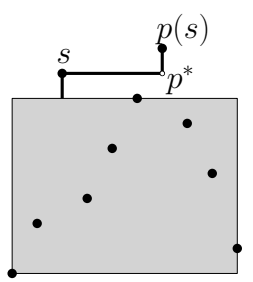

(c)

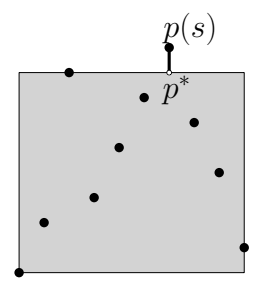

(d)

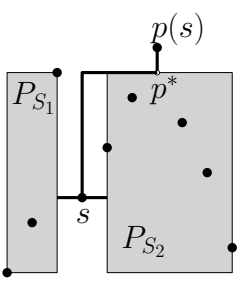

(e)

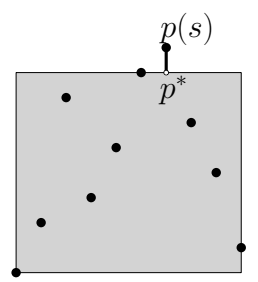

(f)

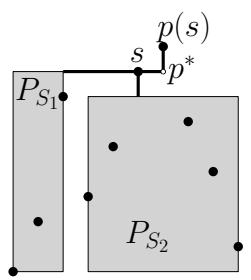

(g)

Fig. 2. Embedding a tree with maximum degree 3 on a set of $n$ points. (a) Embedding $r$. (b)(c) Embedding $s$ with exactly one child. (d)-(g) Embedding $s$ with two children.

directly follows from Theorem 1 Let the points in $P$ be $p_{1}, \ldots, p_{4 n}$ sorted from left to right. Let $P_{2}$ consist of the points $p_{2 i}(1 \leq i \leq 2 n)$ and let $P_{1}=P \backslash P_{2}$. Clearly, the points in $P_{1}$ and $P_{2}$ have the desired horizontal spacing and one of the sets, say $P_{1}$ must contain at least $2 n$ points. Repeating the argument for $P_{1}$ vertically yields the claim.

For trees with maximum degree 3 , we can improve this result.

Theorem 3. Every tree with $n$ vertices and with maximum degree 3 admits a planar orthogeodesic point-set embedding on every general point set with $n$ points.

Proof: Let $T$ be any tree of degree 3 and let $P$ be any general point set with $n$ points. Root $T$ at any leaf $r$. Construct a point-set embedding of $T$ on $P$ as follows. First, embed $r$ on the topmost point $p_{t}$ of $P$ and assign to the subtree $T^{\prime}=T \backslash\{r\}$ point set $P \backslash\left\{p_{t}\right\}$ and an axis-parallel rectangle $R_{T^{\prime}}$ whose opposite corners are the left-bottom corner of the bounding-box of $P$ and the point one unit below the right-top corner of the bounding-box of $P$. Connect $r$ with the top border of $R_{T^{\prime}}$ by drawing a vertical segment from $p_{t}$ to the point $p^{*}$ one unit below $p_{t}$ (see Fig. 2a).

Second, we traverse $T$ top-down. At each step we suppose that a point set $P_{S}$ and an axis-parallel rectangle $R_{S}$ have been assigned to a subtree $S$ of $T$ with root $s$ so that the following invariants are satisfied: (i) $|S|=\left|P_{S}\right|$; (ii) $P_{S}$ lies inside $R_{S}$; (iii) the parent $p(s)$ of $s$ lies outside $R_{S}$ and a horizontal or vertical segment $\overline{p(s), p^{*}}$ has been drawn connecting $p(s)$ to a point $p^{*}$ on the border of $R_{S}$; (iv) let $S$ and $S^{\prime}$ be two subtrees of $T$; if $S$ is contained in $S^{\prime}$, then $R_{S}$ is contained inside $R_{S^{\prime}}$, if $S^{\prime}$ is contained in $S$, then $R_{S^{\prime}}$ is contained inside $R_{S}$, and if neither $S$ is contained in $S^{\prime}$ nor $S^{\prime}$ is contained in $S$, then $R_{S} \cap R_{S^{\prime}}=\emptyset$. Suppose that $p^{*}$ is on the top side of $R_{S}$; the cases in which $p^{*}$ is on the bottom, left, or right side of $R_{S}$ can be discussed analogously. 
If $s$ has exactly one child $s_{1}$, then denote by $S_{1}$ the subtree of $T$ rooted at $s_{1}$. Refer to Figs. $2 \mathrm{~b}-2 \mathrm{c}$ Embed $s$ on the topmost point $p_{t}$ of $P_{S}$, assign to $S_{1}$ the point set $P_{S} \backslash\left\{p_{t}\right\}$ and the rectangle $R_{S_{1}}$ whose opposite corners are the left-bottom corner of $R_{S}$ and the point one unit below the right-top corner of $R_{S}$. Connect $p(s)$ to $s$ by possibly extending $\overline{p(s), p^{*}}$ until its endpoint different from $p(s)$ lies in a point $p_{h}$ on the horizontal line through $p_{t}$ and by drawing a segment $\overline{p_{t}, p_{h}}$. Finally, draw a vertical segment connecting $s$ to the top side of $R_{S_{1}}$. See Fig. 2c

If $s$ has two children $s_{1}$ and $s_{2}$ that are roots of subtrees $S_{1}$ and $S_{2}$, respectively, then denote by $P_{S_{1}}$ (resp. by $P_{S_{2}}$ ) the point set composed of the leftmost $\left|S_{1}\right|$ points of $P_{S}$ (resp. the rightmost $\left|S_{2}\right|$ points of $P_{S}$ ). Denote by $p$ the only point of $P_{S}$ that is neither in $P_{S_{1}}$ nor in $P_{S_{2}}$. Assign to $S_{1}$ the point set $P_{S_{1}}$ and the rectangle $R_{S_{1}}$ whose opposite corners are the left-bottom corner of $R_{S}$ and the intersection point between the top side of $R_{S}$ and the vertical line one unit to the left of $p$. Consider the horizontal segment $h$ which lies on the same horizontal line of the top side of $P_{S}$ and whose endpoints $p_{1}$ and $p_{2}$ lie on the vertical lines through $p$ and through $p(s)$, respectively. We distinguish two cases. (1) In the first case, $h$ does not contain any point of $P_{S}$ in its interior. Refer to Figs. 2d, 2e Embed $s$ on $p$; assign to $S_{2}$ the point set $P_{S_{2}}$ and the rectangle $R_{S_{2}}$ whose opposite corners are the right-bottom corner of $R_{S}$ and the intersection point between the top side of $R_{S}$ and the vertical line one unit to the right of $p$. Connect $p(s)$ to $s$ with an edge composed of $\overline{p(s), p^{*}}$, of a segment between $p^{*}$ and the intersection point $p^{\prime}$ between the top side of $R_{S}$ and the vertical line through $p$, and of segment $\overline{p^{\prime}, p}$. Finally, draw a horizontal segment connecting $s$ with the right side of $R_{S_{1}}$ and draw a horizontal segment connecting $s$ with the left side of $R_{S_{2}}$. (2) In the second case, $h$ contains a point $t\left(P_{S}\right)$ in its interior. Refer to Figs. 2f $2 \mathrm{~g}$. Embed $s$ on $t\left(P_{S}\right)$; assign to $S_{2}$ the point set $P_{S_{2}} \backslash\left\{t\left(P_{S}\right)\right\} \cup\{p\}$ and the rectangle $R_{S_{2}}$ whose opposite corners are the right-bottom corner of $R_{S}$ and the intersection point between the horizontal line one unit below the top side of $R_{S}$ and the vertical line through $p$. Connect $p(s)$ to $s$ with an edge composed of $\overline{p(s), p^{*}}$ and of segment $\overline{p^{*}, t\left(P_{S}\right)}$. Finally, draw a horizontal segment connecting $s$ with the right side of $R_{S_{1}}$ and draw a vertical segment connecting $s$ with the top side of $R_{S_{2}}$.

The only drawn edge $(p(s), s)$ is an orthogeodesic edge. Hence, the resulting drawing is an orthogeodesic point-set embedding of $T$ on $P$. Moreover, it is easy to see that the invariants are maintained at every subtree of $T$ and that such invariants imply the planarity of the point-set embedding. The statement of the theorem follows.

A caterpillar is a tree such that by removing all leaves we are left with a path, called spine. In Theorem 2 we show that every tree with maximum degree 4 has a planar orthogeodesic point-set embedding on every general point set with $4 n$ points. For caterpillars with maximum degree 4 , however, this result is not tight.

Theorem 4. Every caterpillar with $n$ vertices and with maximum degree 4 admits a planar orthogeodesic point-set embedding on every general point set with $\lfloor 1.5 n\rfloor$ points.

Proof: Let $C$ be any caterpillar with $n$ vertices and degree 4 and let $n_{i}$ denote the number of vertices of $C$ with degree $i=1, \ldots, 4$. Let $P^{*}$ be any point set with $\lfloor 1.5 n\rfloor$ points. From $P^{*}$ we arbitrarily choose a point set $P$ of size $N=n+n_{3}+n_{4}$ points on 
which we embed $C$. First, we show that $N \leq 1.5 n$, which implies $N \leq\lfloor 1.5 n\rfloor$ since $N$ is a natural number. Suppose for contradiction that $n_{3}+n_{4}>n / 2$. Since each vertex with degree at least 3 is incident to a leaf this yields $n_{1} \geq n_{3}+n_{4}$. Summing up we have $n \geq n_{1}+n_{3}+n_{4} \geq 2\left(n_{3}+n_{4}\right)>n$, a contradiction.

Next, we show how to embed $C$ on $P$. Each vertex $v \in V$ is mapped to a point $\pi(v) \in P$. Let $S=\left(u_{1}, \ldots, u_{k}\right)$ be the spine of $C$. Remove from $C$ all the leaves, except for one leaf $u_{0}$ incident to $u_{1}$ and one leaf $u_{k+1}$ incident to $u_{k}$. Denote by $S^{+}$the path $\left(u_{0}, u_{1}, \ldots, u_{k}, u_{k+1}\right)$. For $i=1, \ldots, k$, consider node $u_{i}$. If $u_{i}$ has two adjacent leaves not in $S^{+}$, label one of them by "top" and one of them by "bottom"; if $u_{i}$ has one adjacent leaf not in $S^{+}$, arbitrarily label it by "top" or by "bottom". Let $B$ and $T$ be the sets of leaves of $C$ that have been labeled by bottom and by top, respectively.

Let $P_{T}$ be the subset of the highest $|T|$ points of $P$ and let $P_{B}$ be the subset of the lowest $|B|$ points. Further, let $Q=P \backslash\left(P_{T} \cup P_{B}\right)$ be the remaining points. By construction $Q$ contains $t=n_{2}+2\left(n_{3}+n_{4}\right)+2$ points. We embed $C$ on $P$ as follows: (S1) The leaves in $T$ will be embedded on $P_{T}$, the leaves in $B$ will be embedded on $P_{B}$ and the vertices in $S^{+}$will be embedded on a subset $P_{S^{+}} \subseteq Q$. (S2) The spine will be embedded as an $x$-monotone chain such that $u_{i}$ is left of $u_{i+1}$ for all $0 \leq i \leq k$. (S3) Edge $\left\{u_{i}, u_{i+1}\right\}$ occupies the horizontal segment incident to $u_{i}$ on the right for all $0 \leq i \leq k$. If, additionally, the degree of $u_{i}$ is at least 3 , then edge $\left\{u_{i-1}, u_{i}\right\}$ occupies the horizontal segment incident to $u_{i}$ on the left for all $1 \leq i \leq k$.

Let $q_{1}, \ldots, q_{t}$ be the points in $Q$ sorted from left to right. First, we map $u_{0}$ to the leftmost point $q_{1}$ in $Q$. Suppose, we have mapped $u_{0}, \ldots, u_{i}$ for some $i<k+1$ and let $q_{j}=\pi\left(u_{i}\right)$. If $u_{i+1}$ has degree 2 , then we map $u_{i+1}$ to $q_{j+1}$ and we connect $u_{i}$ and $u_{i+1}$ by an $L$-shaped orthogeodesic chain composed of a horizontal segment incident to $u_{i}$ and a vertical segment incident to $u_{i+1}$. See Figs. 3a and 3b If $u_{i+1}$ has degree at least 3 , then we map $u_{i+1}$ to $q_{j+2}$ skipping the point $q_{j+1}$ in $Q$ and we connect $u_{i}$ by an orthogeodesic chain consisting of two horizontal segments incident to $u_{i}$ and $u_{i+1}$, respectively, and a vertical segment in the column to the left of $q_{j+2}$. See Figs. $3 \mathrm{c}$ and $3 \mathrm{~d}$. By construction, $u_{k+1}$ is mapped to a point $q_{j}$ such that $j \leq n_{2}+2\left(n_{3}+n_{4}\right)+2$ since we only skipped points for vertices with degree at least 3 .

Now we describe how to embed the leaves in $T$ on $P_{T}$. The leaves in $B$ are embedded on $P_{B}$ analogously. Let $w_{1}, \ldots, w_{|T|}$ be the vertices in $T$ sorted such that their corresponding vertices on the spine are sorted from left to right and let $T_{i}$ be the set of vertices in $T$ that are incident to vertices $u_{j}$ for $j<i$. For each $i$ let $P_{i}^{-}$be the set of points in $P_{T}$ to the left of $\pi\left(u_{i}\right)$ and let $P_{i}^{+}$be the set of points in $P_{T}$ to the right of $\pi\left(u_{i}\right)$, respectively. Each leaf $w_{i}$ is mapped to a point $\pi\left(w_{i}\right)$ and is attached to the spine by an $L$-shaped orthogeodesic chain. We maintain the following invariant: (L1) If $w_{i}$ is incident to $u_{j}$ and $\left|P_{j}^{-}\right|>\left|T_{j}\right|$, then $w_{i}$ is mapped to the lowest point $p \in P_{i}^{-} \backslash \bigcup_{l=1}^{i-1}\left\{\pi\left(w_{l}\right)\right\}$ by an $L$-shaped orthogeodesic chain consisting of the vertical segment incident to $\pi\left(u_{j}\right)$ and the horizontal segment incident to $p$. Otherwise, $w_{i}$ is mapped to the highest unused point in $P_{i}^{+} \backslash \bigcup_{l=1}^{i-1}\left\{\pi\left(w_{l}\right)\right\}$. See Figure $3 \mathrm{e}$. The resulting point-set embedding is orthogeodesic by construction. Planarity follows from the invariants as follows.

Due to invariants (S1) and (S2) the spine is mapped to an $x$-monotone chain such that the angle at vertices with degree at least 3 is 180 degrees. This implies that the spine 


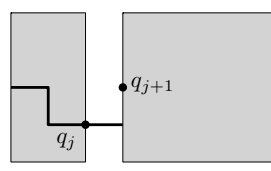

(a)

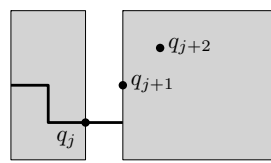

(c)

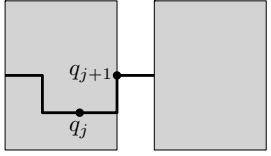

(b)

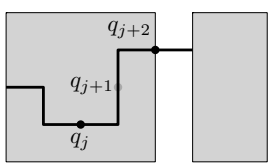

(d)

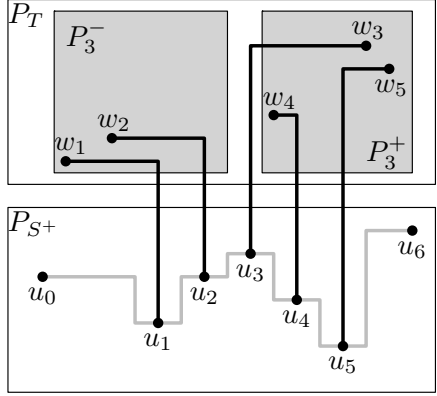

(e)

Fig. 3. Embedding a caterpillar on a set of $\lfloor 1.5 n\rfloor$ points. (a)-(d) Embedding the spine $S^{+}$. (e) Embedding the leaves in $T$.

does not cross itself and that the vertical segments incident to the vertices with degree at least 3 are unoccupied by the spine. Since by invariant (S1) we attached the leaves in $T$ above the spine and the leaves in $B$ below the spine, there cannot be a crossing between two edges incident to a leaf in $T$ and a leaf in $B$, respectively. Suppose for contradiction that there is a crossing between two edges $e_{i}$ and $e_{j}$ incident to two leaves $w_{i}$ and $w_{j}$ in $T$, respectively. Without loss of generality we assume $i<j$. If $\pi\left(w_{i}\right) \in P_{i}^{-}$and $\pi\left(w_{j}\right) \in P_{j}^{+}$there cannot be a crossing by construction. If $\pi\left(w_{i}\right) \in P_{i}^{-} \subseteq P_{j}^{-}$and $\pi\left(w_{j}\right) \in P_{j}^{-}$, then a crossing can only occur if $\pi\left(w_{j}\right) \in P_{i}^{-}$and $\pi\left(w_{j}\right)$ is below $\pi\left(w_{i}\right)$, which contradicts invariant (L1). Analogously, if $\pi\left(w_{i}\right) \in P_{i}^{+}$and $\pi\left(w_{j}\right) \in$ $P_{j}^{+} \subseteq P_{i}^{+}$, then a crossing can only occur if $\pi\left(w_{i}\right) \in P_{j}^{+}$and $\pi\left(w_{i}\right)$ is below $\pi\left(w_{j}\right)$, which contradicts invariant (L1). Finally, if $\pi\left(w_{j}\right) \in P_{i}^{-}$and $\pi\left(w_{i}\right) \in P_{j}^{+} \subseteq P_{i}^{+}$, then this contradicts invariant (L1), since $w_{i}$ is only mapped to a point in $P_{i}^{+}$if there is no unused point in $P_{i}^{-}$. Therefore, the embedding is crossing-free, which concludes the proof.

\section{Planar L-Shaped Orthogeodesic Pointset Embeddings}

Next, we consider planar $L$-shaped orthogeodesic point-set embeddings of trees. First, we prove that every tree with $n$ vertices and with maximum degree 4 admits a planar $L$-shaped point-set embedding on every general point set with $n^{2}-2 n+2$ points. Every point set of this size contains a diagonal point set, which is universal for planar $L$-shaped point-set embeddings of trees with maximum degree 4 . Let $P$ be a point set and let $p_{1}, \ldots, p_{n}$ be the points in $P$ ordered by increasing $x$-coordinates. Then $P$ is diagonal if $y\left(p_{i+1}\right)>y\left(p_{i}\right)$ for every $i=1, \ldots, n-1$ (then $P$ is a positive-diagonal point set), or if $y\left(p_{i+1}\right)<y\left(p_{i}\right)$ for every $i=1, \ldots, n-1$ (then $P$ is a negativediagonal point set). We have the following:

Theorem 5. Every tree with $n$ vertices and with maximum degree 4 admits a planar $L$-shaped point-set embedding on every diagonal point set with $n$ points. 
Proof: We prove by induction a stronger statement. Let $T$ be any tree with $n$ vertices and with maximum degree 4 . Root $T$ in a vertex $r$ of degree at most 3 . We prove that $T$ admits a planar $L$-shaped point-set embedding on every diagonal point set with $n$ points with the further property that there is no edge overlapping or crossing a half-line arbitrarily chosen among the two horizontal half-lines and the two vertical half-lines starting at $r$.

In the base case $n=1$ and the statement is trivially true. In the inductive case, we prove that $T$ admits a planar $L$-shaped point-set embedding on every diagonal point set $P$ with $n$ points with the further property that no edge overlaps or crosses the vertical half-line starting at $r$ and directed upward (the other constructions are analogous). We also suppose that $P$ is a positive-diagonal point set, the case in which it is a negativediagonal point set is analogous. Let $n_{1} \geq 0, n_{2} \geq 0$, and $n_{3} \geq 0$ denote the number of vertices in the subtrees $T_{1}, T_{2}$, and $T_{3}$ rooted at the children $r_{1}, r_{2}$, and $r_{3}$ of $r$, respectively. Let $P_{1}, P_{2}$, and $P_{3}$ be the point sets consisting of the bottommost $n_{1}$ points of $P$, of the bottommost $n_{2}$ points of $P \backslash P_{1}$, and of the topmost $n_{3}$ points of $P$, respectively. Let $p$ be the only point of $P$ not in $P_{1}$, not in $P_{2}$, and not in $P_{3}$. Embed $r$ on $p$. Inductively construct a non-planar $L$-shaped point-set embedding of $T_{1}$ on $P_{1}$ (resp. of $T_{2}$ on $P_{2}$, resp. of $T_{3}$ on $P_{3}$ ) such that no edge overlaps or crosses the vertical line through $r_{1}$ directed upward (resp. the horizontal line through $r_{2}$ directed rightward, resp. the vertical line through $r_{3}$ directed downward). Connect $r$ with $r_{1}$ (resp. with $r_{2}$, resp. with $r_{3}$ ) by an $L$-shaped edge horizontally attached to $r$ and vertically attached to $r_{1}$ (resp. vertically attached to $r$ and horizontally attached to $r_{2}$, resp. horizontally attached to $r$ and vertically attached to $r_{3}$ ). Since the embeddings of $T_{1}$ on $P_{1}$, of $T_{2}$ on $P_{2}$, and of $T_{3}$ on $P_{3}$ are $L$-shaped, all the edges of $T_{1}$, all the edges of $T_{2}$, and all the edges of $T_{3}$ lie inside the bounding boxes of $P_{1}$, of $P_{2}$, and of $P_{3}$, respectively. Hence, the vertical half-line through $r$ directed upward has no overlapping or crossing edge, completing the induction.

According to the Erdős-Szekeres theorem [8], every general point set with $n^{2}-2 n+2$ points contains either a positive-diagonal point set with $n$ points or a negative-diagonal point set with $n$ points. Hence, from Theorem 5 we have the following theorem.

Theorem 6. Every tree with $n$ vertices and with maximum degree 4 admits a planar $L$-shaped point-set embedding on every general point set with $n^{2}-2 n+2$ points.

For caterpillars with maximum degree 4 we can improve the bound of Theorem 6 as follows:

Theorem 7. Every caterpillar with $n$ vertices and with maximum degree 4 admits a planar L-shaped point-set embedding on every general point set with $3 n-2$ points.

Proof: We prove by induction a stronger statement. Let $C$ be any caterpillar with $n$ vertices and with maximum degree 4 . Let $\left(u_{2}, \ldots, u_{k-1}\right)$ be the spine of $C$. Let $u_{1}$ and $u_{k}$ be two leaves of $C$ adjacent to $u_{2}$ and to $u_{k-1}$, respectively. For any $i=1, \ldots, k-1$ denote by $C_{i}$ the subtree of $C$ induced by nodes $u_{1}, \ldots, u_{i}$ and by their adjacent leaves in $C-u_{k}$ and denote $C_{k}:=C$. Observe that $C_{i}$ is a caterpillar, for $i=1, \ldots, k$. We will prove that, for $i=1, \ldots, k, C_{i}$ admits a planar $L$-shaped point-set embedding on every general point set with $3\left|C_{i}\right|-2$ points, so that the following invariant is satisfied: 


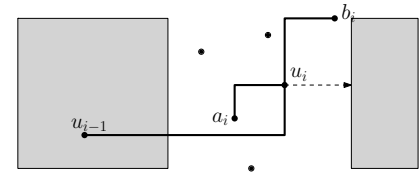

(a)

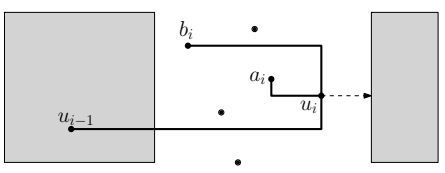

(b)

Fig. 4. Planar $L$-shaped point-set embedding of caterpillars on general point sets. (a) $y\left(p_{1}\right)<$ $y\left(p_{2}\right)<y\left(p_{3}\right)$. (b) $y\left(p_{1}\right)>y\left(p_{2}\right)>y\left(p_{3}\right)$.

The horizontal half-line starting at $u_{i}$ directed rightward does not intersect any edge of the constructed drawing of $C_{i}$. We use the fact that $(\star)$ every general point set with 5 points contains a diagonal point set with 3 points, which can easily be verified.

We now prove the statement. In the base case, $i=1$. Then $\left|C_{1}\right|=1$ and the statement is trivially true. Suppose the statement is true for $C_{i-1}$. Consider any point set $P_{i}$ with $3\left|C_{i}\right|-2$ points. Denote by $P_{i-1}$ the point set consisting of the leftmost $3\left|C_{i-1}\right|-2$ points of $P_{i}$. Construct, by induction, a planar $L$-shaped point-set embedding of $C_{i-1}$ on $P_{i-1}$ so that the horizontal half-line starting at $u_{i-1}$ directed rightward does not intersect any edge of the constructed drawing of $C_{i-1}$. We distinguish three cases.

In the first case $u_{i}$ has no adjacent leaf. Then, embed $u_{i}$ on the rightmost point of $P_{i}$ (such a point exists since $\left|P_{i} \backslash P_{i-1}\right|=3$ ). Connect $u_{i}$ with $u_{i-1}$ by an $L$-shaped edge horizontally attached to $u_{i-1}$ and vertically attached to $u_{i}$.

In the second case $u_{i}$ has one adjacent leaf $a_{i}$. Then, consider the three leftmost points of $P_{i} \backslash P_{i-1}$ (such points exist since $\left|P_{i} \backslash P_{i-1}\right|=6$ ). Then, either two of such three points are above the horizontal line $h\left(u_{i-1}\right)$ through $u_{i-1}$ or two are below. Suppose two points $p_{1}$ and $p_{2}$ are above $h\left(u_{i-1}\right)$, the other case being analogous. Then, embed $u_{i}$ on the rightmost of $p_{1}$ and $p_{2}$ and embed $a_{i}$ on the leftmost of $p_{1}$ and $p_{2}$. Connect $u_{i}$ with $u_{i-1}$ by an $L$-shaped edge horizontally attached to $u_{i-1}$ and vertically attached to $u_{i}$ and connect $u_{i}$ with $a_{i}$ by an $L$-shaped edge horizontally attached to $u_{i}$ and vertically attached to $a_{i}$.

In the third case $u_{i}$ has two adjacent leaves $a_{i}$ and $b_{i}$. Then, consider the nine leftmost points of $P_{i} \backslash P_{i-1}$ (such points exist since $\left|P_{i} \backslash P_{i-1}\right|=9$ ). Then, either five of such nine points are above the horizontal line $h\left(u_{i-1}\right)$ through $u_{i-1}$ or five are below. Suppose five points $p_{1}, \ldots, p_{5}$ are above $h\left(u_{i-1}\right)$, the other case being analogous. Then, by $(\star)$, three points, say without loss of generality $p_{1}, p_{2}$, and $p_{3}$, form a diagonal point set. Suppose, without loss of generality, that $x\left(p_{1}\right)<x\left(p_{2}\right)<x\left(p_{3}\right)$. Then, if $y\left(p_{1}\right)<y\left(p_{2}\right)<y\left(p_{3}\right)$ (see Fig. 4a) embed $u_{i}$ on $p_{2}$, embed $a_{i}$ on $p_{1}$, and embed $b_{i}$ on $p_{3}$; otherwise, that is $y\left(p_{1}\right)>y\left(p_{2}\right)>y\left(p_{3}\right)$ (see Fig. 4b), embed $u_{i}$ on $p_{3}$, embed $a_{i}$ on $p_{2}$, and embed $b_{i}$ on $p_{1}$. In both cases, connect $u_{i}$ with $u_{i-1}$ by an $L$-shaped edge horizontally attached to $u_{i-1}$ and vertically attached to $u_{i}$, connect $u_{i}$ with $a_{i}$ by an $L$-shaped edge horizontally attached to $u_{i}$ and vertically attached to $a_{i}$, and connect $u_{i}$ with $b_{i}$ by an $L$-shaped edge vertically attached to $u_{i}$ and horizontally attached to $b_{i}$.

Since the planar $L$-shaped point-set embedding of $C_{i-1}$ on $P_{i-1}$ satisfies the invariant, the resulting $L$-shaped point-set embedding of $C_{i}$ on $P_{i}$ is planar. Moreover, such a point-set embedding clearly satisfies the invariant, thus completing the induction. 


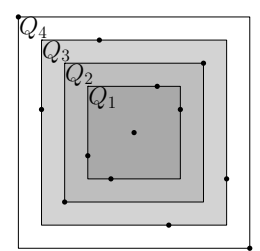

(a)

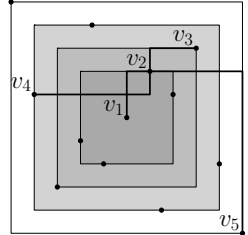

(b)

Fig. 5. Non-planar $L$-shaped point-set embedding of a tree with maximum degree 4

For caterpillars with maximum degree 3 we can improve this bound even further by showing that every such a caterpillar can be embedded on every general point set with $n$ points using $L$-shaped edges. The proof of the following Theorem 8 is based on an inductive argument showing that we can embed a sub-caterpillar $C_{i}$ of $C$, where $C_{i}$ is defined according to the proof of Theorem 7 , on the leftmost $\left|C_{i}\right|$ points of $P$ such that, the horizontal half-line as well as one vertical half-line starting at the rightmost vertex of the spine of $C_{i}$ do not intersect the drawing of $C_{i}$. A proof can be found in [11].

Theorem 8. Every caterpillar with $n$ vertices and with maximum degree 3 admits a planar L-shaped point-set embedding on every general point set with $n$ points.

\section{Non-Planar $L$-Shaped Orthogeodesic Point-Set Embeddings}

In this section we consider non-planar $L$-shaped orthogeodesic point-set embeddings. We start by showing that every tree with $n$ vertices as a non-planar $L$-shaped orthogeodesic point-set embedding on every general point set with $4 n-3$ points.

Theorem 9. Every tree with $n$ vertices and with maximum degree 4 admits a nonplanar L-shaped point-set embedding on every general point set with $4 n-3$ points.

Proof: Let $T=(V, E)$ be a tree with $n$ vertices and let $P$ be a point set with $4 n-3$ points. Let $T$ be rooted in a leaf $r \in V$ and let the vertices of $T$ be labeled $r=$ $v_{1}, \ldots, v_{n}$ according to a depth-first search in $T$. Let $Q_{n}=P$. For $n \geq i \geq 1$, let $P_{i}$ consist of the points on the bounding box of $Q_{i}$, and for $n \geq i \geq 2$ let $Q_{i-1}=Q_{i} \backslash P_{i}$. By construction each $P_{i}$ contains at least two and at most four vertices, except for $P_{1}$, which contains at least one vertex. See Figure 5 a

We embed $T$ using $L$-shaped orthogeodesic chains such that vertex $v_{i}$ is mapped to a point in $P_{i}$ for all $1 \leq i \leq n$. We start by mapping the root $v_{1}$ to an arbitrary point $p^{*} \in P_{1}$. Suppose we have embedded all vertices $v_{1}, \ldots v_{i}$ for some $i \geq 1$ and we would like to embed $v_{i+1}$. Since the vertices are ordered according to a depth-first search, we have already embedded the parent $v_{j}$ of $v_{i+1}$. Without loss of generality we may assume that the vertical segment above $v_{j}$ is unoccupied (otherwise we can rotate the instance accordingly). By construction the points in $P_{i}$ are on the bounding box of $Q_{i+1}$, which contains $Q_{i}$ in its interior. Hence, $P_{i}$ contains a point $p_{t}$ above $f\left(v_{j}\right)$. We map $v_{i+1}$ to $p_{t}$ and connect it to $v_{j}$ by a vertical segment incident to $v_{j}$ and the horizontal segment incident to $p_{t}$. See Figure $5 \mathrm{~b}$ 
We show that a point set of size $n$ suffices for all trees with $n$ vertices and maximum degree 3 .

Theorem 10. Every tree with $n$ vertices and with maximum degree 3 admits a nonplanar L-shaped point-set embedding on every general point set with $n$ points.

Proof: We prove by induction a stronger statement. Let $T$ be any tree with $n$ vertices and with maximum degree 3 . Let $r$ be the root of $T$. We prove that $T$ admits a nonplanar $L$-shaped point-set embedding on every general point set with $n$ points with the further property that there is no edge overlapping a line arbitrarily chosen among the horizontal line and the vertical line through $r$.

In the base case $n=1$ and the statement is trivially true. In the inductive case, we prove that $T$ admits a non-planar $L$-shaped point-set embedding on every general point set $P$ with $n$ points with the further property that no edge overlaps the vertical line through $r$ (the construction providing that no edge overlaps the horizontal line through $r$ is analogous). Refer to Fig 6 Let $n_{1} \geq 0$ and $n_{2} \geq 0$ denote the number of vertices in the subtrees $T_{1}$ and $T_{2}$ rooted at the children $r_{1}$ and $r_{2}$ of $r$, respectively. Let $P_{1}$ and $P_{2}$ be the point sets consisting of the leftmost $n_{1}$ and the rightmost $n_{2}$ points of $P$, respectively. Let $p$ be the only point of $P$ not in $P_{1}$ and not in $P_{2}$. Embed $r$ on $p$. Inductively construct a non-planar $L$-shaped point-

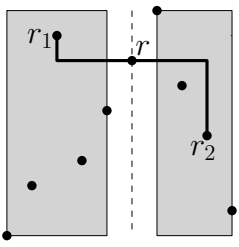

Fig. 6. Non-planar $L$-shaped point-set embedding of a tree set embedding of $T_{1}$ on $P_{1}$ (resp. of $T_{2}$ on $P_{2}$ ) such that no edge overlaps the vertical line through $r_{1}$ (resp. through $r_{2}$ ). Connect $r$ with $r_{1}$ (resp. with $r_{2}$ ) by an $L$-shaped edge horizontally attached to $r$ and vertically attached to $r_{1}$ (resp. to $r_{2}$ ). Since the embedding of $T_{1}$ (resp. of $T_{2}$ ) on $P_{1}$ (resp. on $P_{2}$ ) is $L$-shaped, all the edges of $T_{1}$ (resp. of $T_{2}$ ) lie inside the bounding box of $P_{1}$ (resp. of $P_{2}$ ). Hence, the vertical line through $r$ has no overlapping edge, thus completing the induction.

For caterpillars with maximum degree 4 we can improve this by showing that $n+1$ points suffice for non-planar $L$-shaped orthogeodesic point-set embeddings. The proof is based on an inductive argument showing that every caterpillar has a non-planar $L$ shaped point-set embedding on every general point set such that the spine is embedded as an $x$-monotone chain with the property that each vertex of the spine has a spine edge incident to the right and such that all except possibly one point are used either by the spine or one of its adjacent leaves. The case analysis for the induction can be found in [11].

Theorem 11. Every caterpillar with $n$ vertices and with maximum degree 4 admits a non-planar L-shaped orthogeodesic point-set embedding on every general point set with $n+1$ points.

\section{Conclusions}

In this paper we studied orthogeodesic point-set embeddings of trees on the grid. For various types of drawings $\mathcal{D}$ and various families of trees $\mathcal{F}$ we proved upper bounds 
on the minimum value $f(n)$ such that every $n$-vertex tree in $\mathcal{F}$ admits a point-set embedding of type $\mathcal{D}$ on every point set of size $f(n)$. Since $n$ is a trivial lower bound for $f(n)$ in all considered variants of the problem and since the upper bounds we provided are larger than $n$ for some of the considered variants, it is an interesting topic for future research to close the gap between $n$ and $f(n)$. The gap is especially large for planar $L$-shaped point-set embeddings of trees for which we only proved a quadratic upper bound. Hence it would be interesting to come up with a sub-quadratic upper bound or a non-trivial lower bound. Further, we restricted our attention to trees, but we may consider the same problem for different classes of graphs.

\section{References}

1. Badent, M., Di Giacomo, E., Liotta, G.: Drawing colored graphs on colored points. Theoretical Computer Science 408(2-3), 129-142 (2008)

2. Bose, P.: On embedding an outer-planar graph on a point set. Computational Geometry: Theory and Applications 23, 303-312 (2002)

3. Bose, P., McAllister, M., Snoeyink, J.: Optimal algorithms to embed trees in a point set. Journal of Graph Algorithms and Applications 2(1), 1-15 (1997)

4. Brandenburg, F.J.: Drawing planar graphs on $\frac{8}{9} n^{2}$ area. Electronic Notes in Discrete Mathematics 31, 37-40 (2008)

5. Cabello, S.: Planar embeddability of the vertices of a graph using a fixed point set is NP-hard. Journal of Graph Algorithms and Applications 10(2), 353-366 (2006)

6. Di Giacomo, E., Grilli, L., Krug, M., Liotta, G., Rutter, I.: Hamiltonian Orthogeodesic Alternating Paths. In: Iliopoulos, C.S. (ed.) IWOCA 2011. LNCS, vol. 7056, pp. 170-181. Springer, Heidelberg (2011)

7. Di Giacomo, E., Liotta, G., Trotta, F.: Drawing colored graphs with constrained vertex positions and few bends per edge. Algorithmica 57, 796-818 (2010)

8. Erdős, P., Szekeres, G.: A combinatorial problem in geometry. Compositio Mathematica 2, 463-470 (1935)

9. Everett, H., Lazard, S., Liotta, G., Wismath, S.: Universal sets of $n$ points for one-bend drawings of planar graphs with $n$ vertices. Discrete and Computational Geometry 43, 272 288 (2010)

10. Fink, M., Haunert, J.-H., Mchedlidze, T., Spoerhase, J., Wolff, A.: Drawing graphs with vertices at specified positions and crossings at large angles. pre-print, arXiv:1107.4970v1 (July 2011)

11. Di Giacomo, E., Frati, F., Fulek, R., Grilli, L., Krug, M.: Orthogeodesic point-set embedding of trees. Technical Report 2011-24, Kalrsruhe Institute of Technology, KIT (2011)

12. Gritzmann, P., Mohar, B., Pach, J., Pollack, R.: Embedding a planar triangulation with vertices at specified points. Amer. Math. Monthly 98(2), 165-166 (1991)

13. Katz, B., Krug, M., Rutter, I., Wolff, A.: Manhattan-Geodesic Embedding of Planar Graphs. In: Eppstein, D., Gansner, E.R. (eds.) GD 2009. LNCS, vol. 5849, pp. 207-218. Springer, Heidelberg (2010)

14. Kaufmann, M., Wiese, R.: Embedding vertices at points: Few bends suffice for planar graphs. Journal of Graph Algorithms and Applications 6(1), 115-129 (2002)

15. Kurowski, M.: A 1.235 lower bound on the number of points needed to draw all $n$-vertex planar graphs. Information Processing Letters 92(2), 95-98 (2004) 